\title{
ROJ
}

\section{Optimal dose and volume for postoperative radiotherapy in brain oligometastases from lung cancer: a retrospective study}

\author{
Seung Yeun Chung, MD1, Jong Hee Chang, MD, PhD², Hye Ryun Kim, MD, PhD ${ }^{3}$, \\ Byoung Chul Cho, MD, PhD³ ${ }^{3}$ Chang Geol Lee, MD, PhD', Chang-Ok Suh, MD, PhD ${ }^{1}$ \\ 'Department of Radiation Oncology, Yonsei Cancer Center, Yonsei University College of Medicine, Seoul; \\ ${ }^{2}$ Department of Neurosurgery, Severance Hospital, Yonsei University College of Medicine, Seoul; \\ ${ }^{3}$ Department of Internal Medicine, Yonsei Cancer Center, Yonsei University College of Medicine, Seoul, Korea
}

\begin{abstract}
Purpose: To evaluate intracranial control after surgical resection according to the adjuvant treatment received in order to assess the optimal radiotherapy (RT) dose and volume.

Materials and Methods: Between 2003 and 2015, a total of 53 patients with brain oligometastases from non-small cell lung cancer (NSCLC) underwent metastasectomy. The patients were divided into three groups according to the adjuvant treatment received: whole brain radiotherapy (WBRT) \pm boost (WBRT \pm boost group, $n=26$ ), local RT/Gamma Knife surgery (local RT group, $n=14$ ), and the observation group $(n=13)$. The most commonly used dose schedule was WBRT (25 Gy in 10 fractions, equivalent dose in 2 Gy fractions [EOD2] 26.04 Gy) with tumor bed boost (15 Gy in 5 fractions, EQD2 16.25 Gy).

Results: The WBRT \pm boost group showed the lowest 1-year intracranial recurrence rate of $30.4 \%$, followed by the local RT and observation groups, at $66.7 \%$, and $76.9 \%$, respectively $(p=0.006)$. In the WBRT \pm boost group, there was no significant increase in the 1-year new site recurrence rate of patients receiving a lower dose of WBRT (EOD2) $<27$ Gy compared to that in patients receiving a higher WBRT dose $(p=0.553)$. The 1-year initial tumor site recurrence rate was lower in patients receiving tumor bed dose (EQD2) of $\geq 42.3 \mathrm{~Gy}$ compared to those receiving $<42.3 \mathrm{~Gy}$, although the difference was not significant $(p=0.347$ ).

Conclusions: Adding WBRT after resection of brain oligometastases from NSCLC seems to enhance intracranial control. Furthermore, combining lower-dose WBRT with a tumor bed boost may be an attractive option.
\end{abstract}

Keywords: Lung neoplasms, Neoplasm metastasis, Adjuvant radiotherapy

\section{Introduction}

The incidence of brain metastasis is rising recently due to increasing survival from advances in systemic therapy and imaging technology such as magnetic resonance imaging.
Among brain metastases, the most common primary site is undoubtedly the lungs, with brain metastases incidence rates ranging from $20 \%$ to $50 \%[1,2]$. Even after the diagnosis of brain metastasis, patients are expected to survive longer than before due to improvements in cancer treatment. Therefore,

Received 01 March 2017, Revised 20 March 2017, Accepted 24 March 2017.

Correspondence: Chang-Ok Suh, MD, PhD, Department of Radiation Oncology, Yonsei Cancer Center, Yonsei University College of Medicine, 50-1 Yonsei-ro, Seodaemun-gu, Seoul 03722, Korea. Tel: +82-2-2228-8117, Fax: +82-2-312-9033, E-mail: COSUH317@yuhs.ac

(c) This is an Open Access article distributed under the terms of the Creative Commons Attribution Non-Commercial License (http://creativecommons.org/ licenses/by-nc/4.0/) which permits unrestricted non-commercial use, distribution, and reproduction in any medium, provided the original work is properly cited.

www.e-roj.org 
it is imperative to choose the optimal adaptive treatment modality with the least neurotoxicity. Furthermore, the longer expected survival times make it increasingly important to improve intracranial control and reduce neurotoxicity, especially in patients with brain oligometastases [3].

Surgical resection is recommended in oligometastases for local control and symptom palliation [4]. After metastasectomy, the various adjuvant treatment options include whole brain radiotherapy (WBRT), local radiotherapy (RT) to the tumor bed, or observation with best supportive care. Traditionally, WBRT has been commonly performed, with a typical dose of $30 \mathrm{~Gy}$ in 10 fractions. Even though numerous studies have tried to determine the optimal dose and fractionation for WBRT, it remains controversial [5-9]. There is no doubt of the effect of WBRT on intracranial control, but there has been an increasing concern for the adverse effect of WBRT on neurocognitive function $[10,11]$. Since WBRT neurotoxicity is associated with the total dose and fractionation, lower adjuvant WBRT doses with a boost to the tumor bed and the residual tumor have been performed in our institution in order to decrease neurotoxicity and to ensure adequate tumor bed control [1214]. As the prognosis of brain metastases varies according to the primary tumor site, a study regarding the proper treatment of brain metastases should be performed in a homogenous group of patients with the same primary tumor [3]. The development of targeted therapy has improved survival, especially in non-small cell lung cancer (NSCLC), which has increased the importance of choosing the optimal treatment modality for brain metastasis. Thus, this study focused only on brain oligometastases from NSCLC.

The purpose of this study was to compare the intracranial control rates of WBRT \pm boost $R T$, local $R T$, and observation after the surgical resection of metastatic brain lesions from NSCLC. In addition, the efficacy of a lower WBRT dose with a boost to the tumor bed and residual tumor was also investigated.

\section{Materials and Methods}

\section{Patients}

We retrospectively reviewed the medical records of 130 patients with 1 to 4 brain metastases of primary NSCLC who received metastasectomy between November 2003 and June 2015 at a single institution. Patients who previously received WBRT $(n=22)$, died after surgery $(n=2)$, died or moved to hospice care during RT ( $n=2)$, or had no follow-up imaging after surgery $(n=51)$ were excluded. The remaining 53 patients were divided into three groups according to the adjuvant treatment received. The WBRT \pm boost group $(n=26)$ included patients who received WBRT alone or WBRT with tumor bed boost. The local RT group ( $n=14)$ included patients who received local RT or Gamma Knife surgery (GKS) without WBRT. Local RT was defined as RT to the tumor bed with a suitable margin. The observation group $(n=13)$ included patients who were closely observed without RT. Brain metastases diagnosed with primary NSCLC at the time of diagnosis were defined as synchronous. Patients defined as having a 'new' brain metastasis diagnosis were those who were diagnosed with brain metastasis for the first time, while 'recurrent' included patients who had a previous history of brain metastasis. For 'recurrent' brain metastases patients, previous treatment modalities were GKS 3 times for one patient and GKS once for 3 patients.

\section{Treatment}

All patients complained of symptoms due to brain metastasis prior to surgery. Surgery was performed mainly to resect the symptom-provoking lesion and other metastatic lesions if possible. In terms of the extent of the surgical resection gross total resection (GTR) was defined as the removal of all metastatic lesions, while residual was defined as the subtotal removal of the main mass or the presence of other brain metastatic lesions. GTR was achieved in 100\% of patients with single lesions and in $3.7 \%$ of patients with multiple lesions. The median time interval between surgery and the start of postoperative RT was 19 days (range, 2 to 108 days). All patients but one received RT within 37 days. One patient received local RT (GKS) 108 days after surgery due to wound revision and removal of epidural hemorrhage. Postoperative RT doses and volumes were determined according to the physicians' preferences. The total biological effective dose was calculated as equivalent dose in 2 Gy fractions (EOD2: $\alpha$ ) $\beta$ value of 3 for normal tissue $\left[\mathrm{Gy}_{3}\right]$ and 10 for tumor $\left[G y_{10}\right]$ ). The various WBRT and boost dose schedules are shown in Supplementary Table S1. The most commonly used dose schedule was WBRT as $25 \mathrm{~Gy}$ in 10 fractions (EQD2 26.04 Gy) with a tumor bed boost of $15 \mathrm{~Gy}$ in 5 fractions (EQD2 16.25 Gy). Only half of those patients who received WBRT 30 Gy received a tumor bed boost. WBRT was generally administered using 3D-conformal RT, except for 6 patients who received intensity-modulated radiotherapy (IMRT) with a simultaneousintegrated-boost (SIB) to the tumor bed. In the local RT group, 9 patients received GKS $14-15$ Gy prescribed to the 50\% isodose line, 3 patients received hypofractionated RT (25-40 
Gy in 10-15 fractions), and 2 patients received conventional fractionation RT (45 Gy in 25 fractions).

For postoperative systemic treatment, 11 (20.8\%) and 25 (47.2\%) patients received targeted therapy and chemotherapy, respectively. The targeted therapy included gefitinib $(n=5)$, erlotinib $(n=5)$ and afatinib $(n=1)$. The mutational status was checked in 32 patients, which revealed EGFR mutations in 13 patients (24.5\%) and ALK mutations in 1 (1.9\%). For the patients who were in a systemic progressive status, the median time interval between surgery and the start of systemic chemotherapy were: WBRT \pm boost group 21 days (range, 12-62 days), local RT group 20 days (range, 10 to 62 days), and observation group 19.5 days (range 12 to 40 days).

\section{Outcome evaluations}

The median follow-up period was 17.0 months (range, 3.0 to 118 months). Follow-up was performed regularly, at least every 3 months after surgery, by magnetic resonance imaging (MRI). Initial site recurrence (ISR) was defined as initial tumor bed recurrence or progression. New site recurrence (NSR) was defined as intracranial recurrence (IR) beyond the initial tumor bed including leptomeningeal and parenchymal seeding. IR was defined as all intracranial recurrences including ISR and NSR. Overall survival was defined as the time from surgery until death or the last date that the patient was known to be alive. The IR, ISR, and NSR were defined as the time from surgery until the event of recurrence or the date of last followup in our institution.

\section{Statistical analysis}

The cumulative probability of the IR, ISR, NSR, and overall survival rates were calculated by the Kaplan-Meier method and compared by log-rank tests. Univariate and multivariate analyses were performed using Cox regression. Multivariate analysis was performed using a backward elimination approach including all variables. All analyses were performed using IBM SPSS Statistics for Windows, ver 20.0 (IBM, Armonk, NY, USA).

\section{Results}

\section{Patient and tumor characteristics}

The patient and tumor characteristics are shown in Table 1. The median age of all patients was 56 years (range, 37 to 85 years), and most were confirmed as having adenocarcinoma (83.0\%). About half of the patients had single metastasis, with the remaining having multiple metastases. The median maximum size of the metastatic lesion was $41 \mathrm{~mm}$ (range, 22 to $65 \mathrm{~mm}$ ). The patient and tumor characteristics such as gender, pathology, systemic status, synchronicity, time of brain metastasis diagnosis, number of brain metastasis, maximum size, location, surgery, graded prognostic assessment (GPA) score, mutation subtype, and systemic treatment after surgery did not differ among the three groups.

\section{Intracranial recurrence}

The 1 -year IR rate of all patients was $40.6 \%$. In the WBRT \pm boost, local RT, and observation groups, the 1-year IR rates were $30.4 \%, 66.7 \%$, and 76.9\%, respectively ( $p=0.006$ ) (Fig $1 A)$. In univariate analysis, the adverse factors for IR included squamous cell carcinoma or other pathology (hazard ratio $[H R], 3.86 ; 95 \%$ confidence interval $[\mathrm{Cl}], 1.62-9.20 ; \mathrm{p}=0.002)$ recurrent brain metastasis $(\mathrm{HR}, 3.81 ; 95 \% \mathrm{Cl}, 1.27-11.44$; $p=0.017)$, and multiple brain metastases (HR, 2.21; 95\% $\mathrm{Cl}, 1.02-4.78 ; \mathrm{p}=0.044)$. The favorable factors included targeted therapy as a postoperative systemic treatment compared to none ( $\mathrm{HR}, 0.18,95 \% \mathrm{Cl}, 0.04-0.79 ; p=0.023)$ and WBRT \pm boost as a treatment modality when compared to observation ( $\mathrm{HR}, 0.25 ; 95 \% \mathrm{Cl}, 0.10-0.62 ; \mathrm{p}=0.003)$. In multivariate analysis, IR was adversely affected by recurrent brain metastasis ( $\mathrm{HR}, 13.35 ; 95 \% \mathrm{Cl}, 2.60-68.65 ; \mathrm{p}=0.002)$ and beneficially affected by stable $(\mathrm{HR}, 0.14 ; 95 \% \mathrm{Cl}, 0.02-0.97$; $\mathrm{p}=0.046)$ or NED $(\mathrm{HR}, 0.20 ; 95 \% \mathrm{Cl}, 0.05-0.79 ; \mathrm{p}=0.022)$ systemic status compared to progressive systemic status and WBRT \pm boost as treatment modality when compared to observation ( $\mathrm{HR}, 0.23 ; 95 \% \mathrm{Cl}, 0.09-0.63 ; p=0.004$ ) (Table 2). Local RT as a treatment modality compared to observation did not prove to be a significant factor $(\mathrm{HR}, 0.47 ; 95 \% \mathrm{Cl}, 0.19$ $1.17 ; p=0.105)$

\section{New site recurrence}

A total of 29 patients experienced NSR: 22 patients had new parenchymal recurrence, 4 had seeding metastasis, and 3 had both. The 1-year NSR rates were 30.7\%, 67.3\%, and $76.9 \%$ in the WBRT \pm boost, local RT, and observation groups, respectively ( $p=0.006$ ) (Fig. $1 B)$. The results of multivariate analysis were similar to those of IR. NSR was adversely affected by recurrent brain metastasis ( $\mathrm{HR}, 12.79 ; 95 \% \mathrm{Cl}, 2.48-65.92$; $\mathrm{p}=0.002)$ and beneficially affected by stable $(\mathrm{HR}, 0.12 ; 95 \%$ $\mathrm{Cl}, 0.02-0.88 ; \mathrm{p}=0.037)$ or NED $(\mathrm{HR}, 0.19 ; 95 \% \mathrm{Cl}, 0.05-0.76$; $\mathrm{p}=0.019$ ) systemic status compared to progressive status and WBRT \pm boost as a treatment modality when compared to observation ( $\mathrm{HR}, 0.24 ; 95 \% \mathrm{Cl}, 0.09-0.64 ; \mathrm{p}=0.004$ ) (Table 2). In addition, subgroup analysis of the WBRT \pm boost group was 
Table 1. Patient and tumor characteristics

\begin{tabular}{|c|c|c|c|c|c|}
\hline & $\begin{array}{c}\text { Total } \\
(n=53)\end{array}$ & $\begin{array}{l}\text { WBRT } \pm \text { boost } \\
\quad(n=26)\end{array}$ & $\begin{array}{l}\text { Local RT } \\
(n=14)\end{array}$ & $\begin{array}{c}\text { No RT } \\
(n=13)\end{array}$ & p-value \\
\hline Age (yr) & $56(37-85)$ & $56(37-85)$ & $60(37-78)$ & $56(37-80)$ & \\
\hline Sex & & & & & $0.151^{*}$ \\
\hline Male & $37(69.8)$ & $19(73.1)$ & $7(50)$ & $11(84.6)$ & \\
\hline Female & $16(30.2)$ & $7(26.9)$ & $7(50)$ & $2(15.4)$ & \\
\hline Pathology & & & & & $0.148^{*}$ \\
\hline Adenoca & $44(83)$ & $24(92.3)$ & $12(85.7)$ & $8(61.5)$ & \\
\hline $\mathrm{SCCa}$ & $4(7.5)$ & $1(3.8)$ & $1(7.1)$ & $2(15.4)$ & \\
\hline Other & $5(9.4)$ & $1(3.8)$ & $1(7.1)$ & $3(23.1)$ & \\
\hline Systemic status & & & & & $0.839^{*}$ \\
\hline Progression & $39(73.6)$ & $18(69.2)$ & $11(78.6)$ & $10(76.9)$ & \\
\hline Stable & $3(5.7)$ & $1(3.8)$ & $1(7.1)$ & $1(7.7)$ & \\
\hline NED & $11(20.8)$ & 7 (26.9) & $2(14.3)$ & $2(15.4)$ & \\
\hline Synchronous & & & & & 0.894 \\
\hline Synchronous & $34(64.2)$ & $16(61.5)$ & $9(64.3)$ & $9(69.2)$ & \\
\hline Metachronous & $19(35.8)$ & $10(38.5)$ & $5(35.7)$ & $4(30.8)$ & \\
\hline Brain metastasis diagnosis & & & & & $1.000^{*}$ \\
\hline New & $49(92.5)$ & $24(92.3)$ & 13 (92.9) & $12(92.3)$ & \\
\hline Recurrent & $4(7.5)$ & $2(7.7)$ & $1(7.1)$ & $1(7.7)$ & \\
\hline Number of metastasis & & & & & 0.780 \\
\hline Single & $26(49.1)$ & $14(53.8)$ & $6(42.9)$ & $6(46.2)$ & \\
\hline Multiple & $27(50.9)$ & $12(46.2)$ & $8(57.1)$ & $7(53.8)$ & \\
\hline Maximum size of metastasis (mm) & $41(22-65)$ & $42(22-56)$ & $41(24-65)$ & $39(27-64)$ & 0.482 \\
\hline$<40$ & $21(39.6)$ & $9(34.6)$ & $5(35.7)$ & $7(53.8)$ & \\
\hline$\geq 40$ & $32(60.4)$ & $17(65.4)$ & $9(64.3)$ & $6(46.2)$ & \\
\hline Location of metastasis & & & & & $0.817^{*}$ \\
\hline Supratentorial & $32(60.4)$ & $16(61.5)$ & $7(50)$ & $9(69.2)$ & \\
\hline Infratentorial & $8(15.1)$ & $4(15.4)$ & $2(14.3)$ & $2(15.4)$ & \\
\hline Both & $13(24.5)$ & $6(23.1)$ & $5(35.7)$ & $2(15.4)$ & \\
\hline Surgery extent & & & & & 0.780 \\
\hline Residual & $26(49.1)$ & $12(46.2)$ & $8(57.1)$ & $6(46.2)$ & \\
\hline GTR & $27(50.9)$ & $14(53.8)$ & $6(42.9)$ & $7(53.8)$ & \\
\hline GPA & & & & & $0.504^{*}$ \\
\hline $0.0-1.0$ & $8(15.1)$ & $4(15.4)$ & $1(7.1)$ & $3(23.1)$ & \\
\hline $1.5-2.0$ & $14(26.4)$ & $5(19.2)$ & $7(50)$ & $2(15.4)$ & \\
\hline $2.5-3.0$ & $20(37.7)$ & $11(42.3)$ & $4(28.6)$ & $5(38.5)$ & \\
\hline $3.5-4.0$ & $11(20.8)$ & $6(23.1)$ & $2(14.3)$ & $3(23.1)$ & \\
\hline \multicolumn{6}{|l|}{ Mutation subtype } \\
\hline Unknown & $21(39.6)$ & 7 (26.9) & $8(57.1)$ & $6(46.2)$ & $0.280^{*}$ \\
\hline None & $18(3.4)$ & $12(46.2)$ & $3(21.4)$ & $3(23.1)$ & \\
\hline EGFR & $13(24.5)$ & $7(26.9)$ & $3(21.4)$ & $3(23.1)$ & \\
\hline ALK & $1(1.9)$ & $0(0)$ & $0(0)$ & $1(7.7)$ & \\
\hline \multicolumn{6}{|l|}{ Systemic Tx after surgery } \\
\hline None & $17(32.1)$ & $7(26.9)$ & $4(28.6)$ & $6(46.2)$ & $0.699 *$ \\
\hline Targeted Tx & $11(20.8)$ & 7 (26.9) & $2(14.3)$ & $2(15.4)$ & \\
\hline CTx without targeted Tx & $25(47.2)$ & $12(46.2)$ & $8(57.1)$ & $5(38.5)$ & \\
\hline WBRT dose (Gy) (EQD2) & - & $26.04(20.8-39.1)$ & - & - & \\
\hline Tumor bed dose (Gy) (EQD2) & - & $44.3(42.3-50.0)$ & $88.7(26.0-100.0)$ & - & \\
\hline
\end{tabular}

Values are presented as median (range) or number (\%).

Adenoca, adenocarcinoma; SCCa, squamous cell carcinoma; NED, no evidence of disease; GTR, gross total removal; GPA, graded prognostic assessment; Tx, treatment; CTx, chemotherapy; WBRT, whole brain radiotherapy.

* Fisher's exact test. 


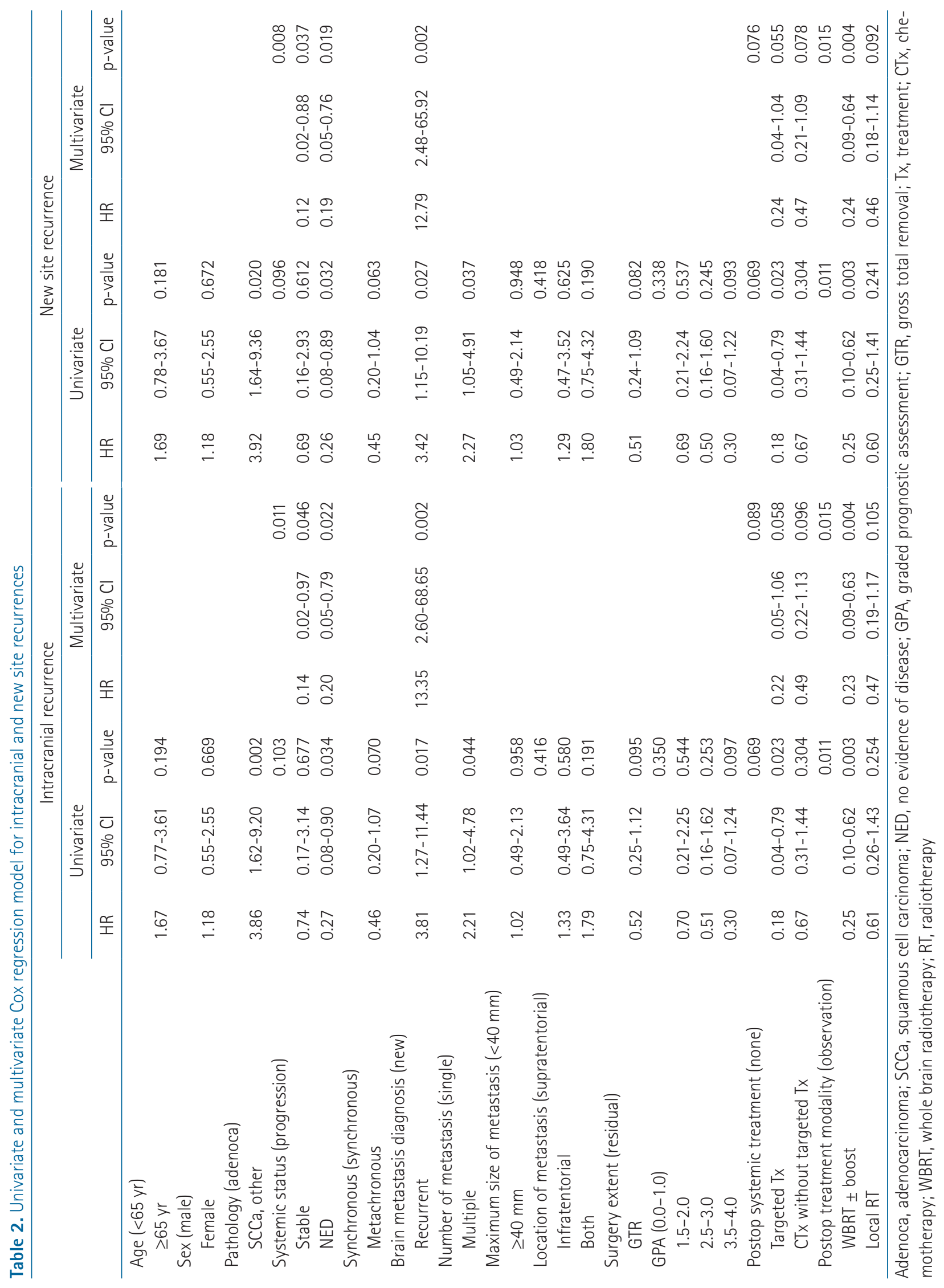


performed in order to investigate the relationship between NSR and WBRT dose. Since the most commonly used dose schedule was WBRT 25 Gy in 10 fractions (EQD2 26.04 Gy), the WBRT \pm boost group was divided into WBRT dose (EQD2) $<27$ Gy $(n=16)$ and $\geq 27$ Gy $(n=10)$ groups. The 1 -year NSR rates were not significantly different between the patients receiving WBRT dose (EQD2) <27 Gy and $\geq 27$ Gy $(40.9 \%$ and 27.3\%, respectively; $p=0.553$ ) (Fig. 2).

\section{Initial site recurrence}

The 1-year ISR rates were 10.3\%, 9.1\%, and $24.8 \%$ in the WBRT \pm boost, local RT, and observation groups, respectively ( $p$ $=0.410$ ) (Fig. 1C). Recurrent brain metastasis (HR, 29.22; 95\% $\mathrm{Cl}, 4.75-179.72 ; \mathrm{p}<0.001)$ proved to be the single adverse factor for ISR by univariate analysis, but not by multivariate analysis. For ISR, subgroup analysis was performed for the WBRT \pm boost and local RT groups combined in order to determine the relationship between ISR and total RT dose delivered to the tumor bed. The subgroup was divided into tumor bed dose (EQD2) <42.3 Gy and $\geq 42.3$ Gy groups because the most commonly used dose schedule was WBRT 25 Gy in 10 fractions (EQD2 $26.04 \mathrm{~Gy}$ ) with a tumor bed boost of $15 \mathrm{~Gy}$ in 5 fractions (EQD2 $16.25 \mathrm{~Gy}$ ), which summed to a total of EQD2 $42.29 \mathrm{~Gy}$ to the tumor bed. The 1-year ISR rate was lower in patients receiving tumor bed dose (EQD2) $\geq 42.29$ Gy compared to those receiving $<42.29$ Gy $(5.0 \%$ vs. $21.2 \%, p=0.347)$, but the difference was not significant (Fig. 3)

\section{Overall survival}

The median overall survival of all patients was 18.7 months. The difference in overall survival among the three groups was not significant ( $p=0.667)$ (Fig. 4). The 1-year survival rates were $79.3 \%, 78.6 \%$, and $69.2 \%$ in the WBRT \pm boost, local RT, and observation groups, respectively. In multivariate analysis, the postoperative treatment modality did not remain a significant factor. Factors such as age older than 65 years, progressive systemic disease status, metachronous brain metastasis, recurrent brain metastasis, metastatic lesion size $\geq 40 \mathrm{~mm}$, and lower GPA score were significant adverse factors for overall survival (Supplementary Table S2).

\section{Discussion and Conclusion}

Currently, there is no solid evidence on the optimal adjuvant treatment after metastasectomy of brain metastasis. All three treatment options-WBRT, local RT, and observation with best supportive care-are used in clinical practice. Thus, this study compared the intracranial control rates of these treatments after surgical resection of metastatic brain lesions from NSCLC. Our results showed that WBRT \pm boost after the resection of a limited number of brain metastases enhanced intracranial control compared to local RT or observation.

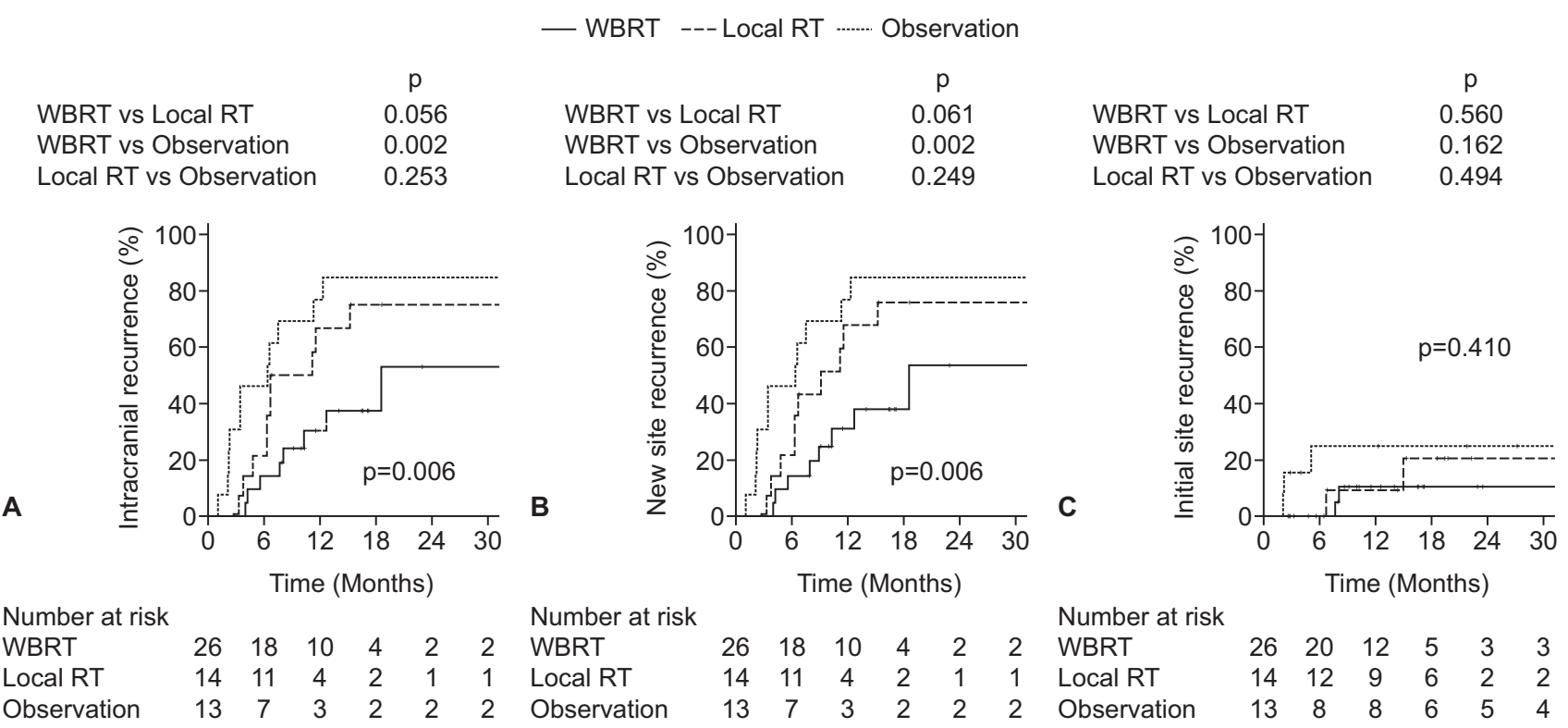

Fig. 1. Kaplan-Meier estimates of intracranial recurrence $(A)$, new site recurrence $(B)$, and initial site recurrence $(C)$, according to the adjuvant treatment received. WBRT, whole brain radiotherapy; RT, radiotherapy. 
It is well known that WBRT enhances intracranial control. A classic randomized study has shown the advantage of WBRT over observation for brain recurrence and neurologic death in patients with single brain metastasis, and in the EORTC 22952 26001 trial, WBRT reduced the 2-year relapse rates at initial (59\% to $27 \%$ ) and new (42\% to 23\%) sites in comparison with those of observation after surgical resection of 1 to 3 brain metastases $[15,16]$. Furthermore, a study comparing WBRT vs. stereotactic radiosurgery (SRS) alone for resected brain metastases reported similar local control rates (83\% vs. 74\%, p $=0.31)$ but higher 1 -year distant brain control for WBRT (70\% vs. $48 \%, p=0.03$ ) [17]. The advantage of WBRT for intracranial control may be explained by the ability to control the increased probability of seeding during surgery $[18,19]$. Thus, local RT may be insufficient for the control NSR, which may explain our findings of no significant difference between the local RT and observation groups for IR and NSR.

As for local control, our results showed similar 1-year ISR rates between the WBRT \pm boost and local RT groups and much higher recurrence rates for the observation group, although the differences were not significant. Other studies have shown similar local control rates. For example, a retrospective study of patients who received SRS (median biological effective dose, $16 \mathrm{~Gy}_{10}$ ) at the postoperative resection cavity (median volume, $7.7 \mathrm{~cm}^{3}$ ) for 1 to 4 brain metastases reported a 1-year control rate of 79\% [20]. Another phase II study showed similar results, with a 1-year local failure of 22\% for SRS (median, 18 Gy) after surgical resection for 1 to 2 brain metastases (median size, $2.9 \mathrm{~cm}$ ) [21]. In addition, a review article reported an estimated 1-year actuarial local control of $85 \%$ for patients who received SRS to the postoperative resection cavity [22] As for overall survival, there were no significant differences among the three groups in this study, which was concordant with the findings of other studies, in which no difference in overall survival was explained by regular follow-up MRI and thus timely salvage treatment $[15,17]$. In addition, since many of the patients had a progressive systemic status, the control of systemic disease may have affected the overall survival.

Since WBRT does not translate into increased survival, there are more reports advocating the use of local RT after surgical resection of brain metastases due to the neurotoxicity of WBRT. A randomized trial to assess neurocognition in patients with brain metastases reported significantly better learning and memory function by 4 months for the SRS alone group compared to that in the SRS plus WBRT group [11]. Thus, a growing number of studies have reported the efficacy of omitting WBRT and delivering local RT after surgical resection for brain oligometastases [20,21]. However randomized trials that directly compare WBRT with local RT after metastasectomy have never been performed. Although RTOG 1270 is ongoing, we will have to wait for some years to see the results $[17,23]$. The present study also investigated
- WBRT EQD2 $\geq 27$ Gy

--- WBRT EQD2 < 27 Gy

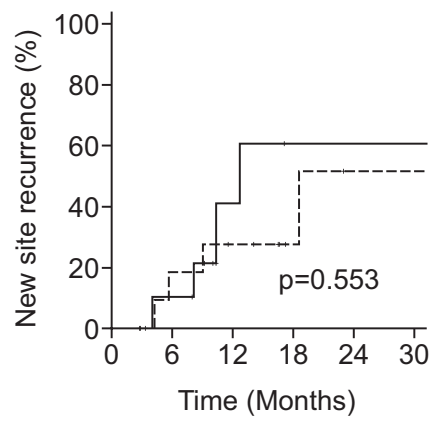

Number at risk

$\geq 27$ Gy

$<27$ Gy

10

$\begin{array}{lllll}9 & 3 & 1 & 1 & 1 \\ 9 & 7 & 3 & 1 & 1\end{array}$

Fig. 2. Kaplan-Meier estimates of new site recurrence according to WBRT dose (EQD2) $\geq 27$ Gy or $<27$ Gy.
- Tumor bed dose EQD2 $\geq 42.29 \mathrm{~Gy}$

--- Tumor bed dose EQD2 < 42.29 Gy

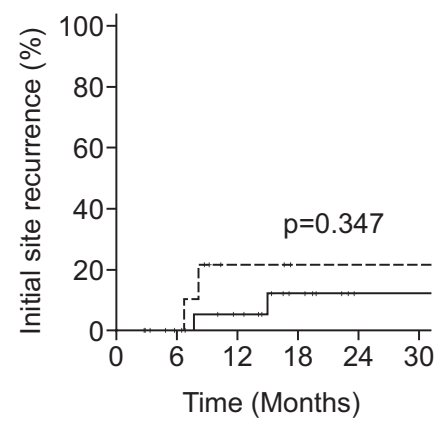

Number at risk

$\begin{array}{lllllll}\geq 42.3 \mathrm{~Gy} & 26 & 22 & 17 & 10 & 4 & 4\end{array}$

$<42.3 \mathrm{~Gy}$

Fig. 3. Kaplan-Meier estimates of initial site recurrence according to tumor bed dose (EOD2) $\geq 42.29$ Gy or $<42.29$ Gy.
— WBRT --- Local RT ....... Observation

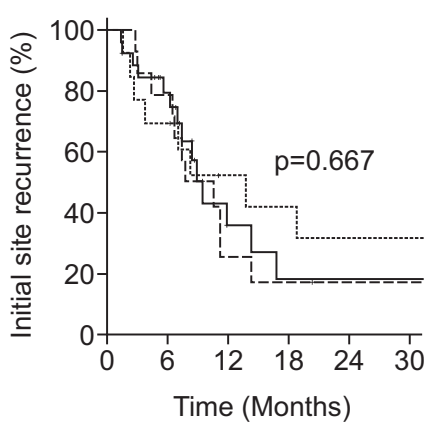

Number at risk

$\begin{array}{lllllll}\text { WBRT } & 26 & 16 & 4 & 2 & 1 & 1\end{array}$

$\begin{array}{lllllll}\text { Local RT } & 14 & 11 & 3 & 2 & 1 & 1\end{array}$ $\begin{array}{lllllll}\text { Observation } & 13 & 9 & 5 & 4 & 3 & 3\end{array}$

Fig. 4. Kaplan-Meier estimates of overall survival according to the adjuvant treatment received. 
a lower WBRT dose with a boost to the tumor bed, since WBRT neurotoxicity is associated with the total dose and fractionation $[12,13]$. Although no studies have directly compared lower WBRT doses to the conventional WBRT dose of $30 \mathrm{~Gy}$, the advantage of lower WBRT dose has been shown in previous studies on prophylactic cranial irradiation (PCI). For example, a French study compared PCl (24 Gy in 8 fractions, EQD2 $26 \mathrm{~Gy}$ ) vs. no $\mathrm{PCl}$ and observed no significant difference between the groups in terms of neuropsychological function or abnormalities [24]. In another trial performed at MD Anderson Cancer Center, 30 patients received PCl (25 Gy in 10 fractions, EQD2 $26.04 \mathrm{~Gy}$ ) and no significant deterioration was observed after PCI [25]. The RTOG 0212 trial compared patients who received lower dose $\mathrm{PCl}(25 \mathrm{~Gy}$ in 10 fractions, EQD2 $26.04 \mathrm{~Gy}$ ) vs. higher dose $\mathrm{PCl}$ (36 Gy in 18 fractions, EQD2 36 Gy or twice-daily 24 fractions, EQD2 $34.50 \mathrm{~Gy}$ ), and showed significant increase of chronic neurotoxicity in the higher dose $\mathrm{PCl}$ group [26]. In addition, with advances in technology, especially hippocampus-sparing IMRT, WBRT with lower neurotoxicity is becoming more feasible [27-29]. Some studies have reported that the hippocampus shows radiation dose-dependent atrophy and that hippocampus-saving WBRT is related with preservation of memory and quality-of-life $[29,30]$. Therefore, if a lower dose of WBRT could control NSR and if a boost RT to the tumor bed could ensure local control, this treatment method may be best. In this study, WBRT $<27$ Gy did not increase NSR in NSCLC patients compared to $>27$ Gy; furthermore, an initial site dose $\geq 42.3$ Gy seemed to show better initial site control rates.

In addition to postoperative treatment modality, another important factor that must be considered for brain metastases is targeted therapy, since targeted agents such as erlotinib or gefitinib are known to penetrate the blood-brain barrier [31,32]. In addition, osimertinib for EGFR T790M-positive lung cancer has shown a benefit in progression-free survival even in patients with brain metastasis [33]. Thus, as some agents are effective in cases of brain metastasis, targeted therapy without RT to the brain is gaining interest, not only for small asymptomatic brain metastases but also for cases of leptomeningeal disease and postoperative brain metastasis [34]. In our study, targeted therapy was a significant beneficial factor for intracranial recurrence compared to no systemic treatment by univariate analysis $(p=0.023)$ and was a borderline beneficial factor by multivariate analysis ( $p=0.058$ ), whereas chemotherapy was not significant when compared to no systemic treatment. However, more patients in the targeted therapy group received WBRT (7 of 11) as compared with chemotherapy group (12 of 25) and no systemic treatment (7 of 17) groups. Currently, the role of targeted therapy in the management of brain metastasis from lung cancer has not been clearly established [34]. The question of whether targeted therapy could replace WBRT in the management of brain metastasis, including the role of controlling the microscopic metastasis such as cerebrospinal fluid contamination from surgical resection, remains to be investigated.

This study has several limitations. Its retrospective nature and patient heterogeneity may have biased the results. In addition, because of the small number of patients, it was difficult to see any significant results in the subgroup analysis and reach any clear conclusions. In addition, no common objective tool was used to assess neurotoxicity. However, this study also has several strengths. First, only postoperative brain oligometastases cases with the same primary cancer origin were included in this study. This is important since the prognosis of brain metastasis varies according to the primary cancer type [3]. Second, this study also investigated the unique idea of a lower WBRT dose with a boost RT to the tumor bed and residual tumor.

In conclusion, WBRT seems to be an essential component of adjuvant treatment after resection considering intracranial control for brain metastases from NSCLC. In addition, lower dose WBRT with a boost to the tumor bed may be an attractive option for both increased tumor control and reduced neurotoxicity. However, further studies are necessary to confirm these findings.

\section{Conflict of Interest}

No potential conflict of interest relevant to this article was reported.

\section{Supplementary Materials}

Supplementary materials can be found via https://doi. org/10.3857/roj.2017.00094. Table S1. WBRT and boost RT dose schedules. Table S2. Univariate and multivariate cox regression model for overall survival.

\section{References}

1. Wen PY, Loeffler JS. Metastatic brain cancer. In: DeVita VT, Hellman S, Rosenberg SA, editors. Cancer: principles and practice of oncology. 6 ed. Philadelphia, PA: Lippincott Williams \& Wilkins; 2001. p. 2655-70. 
2. Barnholtz-Sloan JS, Sloan AE, Davis FG, Vigneau FD, Lai $P$, Sawaya RE. Incidence proportions of brain metastases in patients diagnosed (1973 to 2001) in the Metropolitan Detroit Cancer Surveillance System. J Clin Oncol 2004;22:2865-72.

3. Nussbaum ES, Djalilian HR, Cho KH, Hall WA. Brain metastases: histology, multiplicity, surgery, and survival. Cancer 1996;78:1781-8.

4. Tsao MN, Rades D, Wirth $A$, et al. Radiotherapeutic and surgical management for newly diagnosed brain metastasis(es): An American Society for Radiation Oncology evidence-based guideline. Pract Radiat Oncol 2012;2:210-25.

5. Borgelt B, Gelber R, Kramer $S$, et al. The palliation of brain metastases: final results of the first two studies by the Radiation Therapy Oncology Group. Int J Radiat Oncol Biol Phys 1980;6:1-9.

6. Graham PH, Bucci J, Browne L. Randomized comparison of whole brain radiotherapy, $20 \mathrm{~Gy}$ in four daily fractions versus 40 Gy in 20 twice-daily fractions, for brain metastases. Int J Radiat Oncol Biol Phys 2010;77(3):648-54.

7. Haie-Meder C, Pellae-Cosset B, Laplanche A, et al. Results of a randomized clinical trial comparing two radiation schedules in the palliative treatment of brain metastases. Radiother Oncol 1993;26:111-6.

8. Murray KJ, Scott C, Greenberg HM, et al. A randomized phase III study of accelerated hyperfractionation versus standard in patients with unresected brain metastases: a report of the Radiation Therapy Oncology Group (RTOG) 9104. Int J Radiat Oncol Biol Phys 1997;39:571-4.

9. Priestman TJ, Dunn J, Brada M, Rampling R, Baker PG. Final results of the Royal College of Radiologists' trial comparing two different radiotherapy schedules in the treatment of cerebral metastases. Clin Oncol (R Coll Radiol) 1996;8:308-15.

10. Tallet AV, Azria D, Barlesi $F$, et al. Neurocognitive function impairment after whole brain radiotherapy for brain metastases: actual assessment. Radiat Oncol 2012;7:77.

11. Chang EL, Wefel JS, Hess KR, et al. Neurocognition in patients with brain metastases treated with radiosurgery or radiosurgery plus whole-brain irradiation: a randomised controlled trial. Lancet Oncol 2009;10:1037-44.

12. Aoyama $H$, Tago $M$, Kato $N$, et al. Neurocognitive function of patients with brain metastasis who received either whole brain radiotherapy plus stereotactic radiosurgery or radiosurgery alone. Int J Radiat Oncol Biol Phys 2007;68:1388-95.

13. Filley CM, Kleinschmidt-DeMasters BK. Toxic leukoencephalopathy. N Engl J Med 2001;345:425-32.

14. Ha B, Chung SY, Kim YJ, et al. Effects of postoperative radiotherapy on leptomeningeal carcinomatosis or dural metastasis after resection of brain metastases in breast cancer patients. Cancer Res Treat 2016 0ct 31 [Epub]. http://doi. org/10.4143/crt.2016.303.

15. Kocher $M$, Soffietti $R$, Abacioglu U, et al. Adjuvant whole-brain radiotherapy versus observation after radiosurgery or surgical resection of one to three cerebral metastases: results of the EORTC 22952-26001 study. J Clin Oncol 2011;29:134-41.

16. Patchell RA, Tibbs PA, Regine WF, et al. Postoperative radiotherapy in the treatment of single metastases to the brain: a randomized trial. JAMA 1998;280:1485-9.

17. Patel KR, Prabhu RS, Kandula S, et al. Intracranial control and radiographic changes with adjuvant radiation therapy for resected brain metastases: whole brain radiotherapy versus stereotactic radiosurgery alone. J Neurooncol 2014;120:65763.

18. Atalar B, Modlin LA, Choi CY, et al. Risk of leptomeningeal disease in patients treated with stereotactic radiosurgery targeting the postoperative resection cavity for brain metastases. Int J Radiat Oncol Biol Phys 2013;87:713-8.

19. Jo KI, Lim DH, Kim ST, et al. Leptomeningeal seeding in patients with brain metastases treated by gamma knife radiosurgery. J Neurooncol 2012;109:293-9.

20. Soltys SG, Adler JR, Lipani JD, et al. Stereotactic radiosurgery of the postoperative resection cavity for brain metastases. Int J Radiat Oncol Biol Phys 2008;70:187-93.

21. Brennan C, Yang TJ, Hilden $P$, et al. A phase 2 trial of stereotactic radiosurgery boost after surgical resection for brain metastases. Int J Radiat Oncol Biol Phys 2014;88:130-6.

22. Roberge D, Parney I, Brown PD. Radiosurgery to the postoperative surgical cavity: who needs evidence? Int Radiat Oncol Biol Phys 2012;83:486-93.

23. Kalkanis SN, Kondziolka D, Gaspar LE, et al. The role of surgical resection in the management of newly diagnosed brain metastases: a systematic review and evidence-based clinical practice guideline. J Neurooncol 2010;96:33-43.

24. Arriagada R, Le Chevalier T, Borie F, et al. Prophylactic cranial irradiation for patients with small-cell lung cancer in complete remission. J Natl Cancer Inst 1995;87:183-90.

25. Komaki R, Meyers CA, Shin DM, et al. Evaluation of cognitive function in patients with limited small cell lung cancer prior to and shortly following prophylactic cranial irradiation. Int $\rfloor$ Radiat Oncol Biol Phys 1995;33:179-82.

26. Wolfson AH, Bae $K_{1}$ Komaki $R$, et al. Primary analysis of a phase II randomized trial Radiation Therapy Oncology Group (RTOG) 0212: impact of different total doses and schedules of prophylactic cranial irradiation on chronic neurotoxicity and quality of life for patients with limited-disease small-cell lung 
cancer. Int J Radiat Oncol Biol Phys 2011;81:77-84.

27. Kim KH, Cho BC, Lee CG, et al. Hippocampus-Sparing WholeBrain Radiotherapy and Simultaneous Integrated Boost for Multiple Brain Metastases From Lung Adenocarcinoma: Early Response and Dosimetric Evaluation. Technol Cancer Res Treat 2016;15:122-9.

28. Kundapur V, Ellchuk T, Ahmed S, Gondi V. Risk of hippocampal metastases in small cell lung cancer patients at presentation and after cranial irradiation: a safety profile study for hippocampal sparing during prophylactic or therapeutic cranial irradiation. Int J Radiat Oncol Biol Phys 2015;91:781-6.

29. Gondi V, Pugh SL, Tome WA, et al. Preservation of memory with conformal avoidance of the hippocampal neural stemcell compartment during whole-brain radiotherapy for brain metastases (RTOG 0933): a phase II multi-institutional trial. J Clin Oncol 2014;32:3810-6.

30. Seibert TM, Karunamuni $R$, Bartsch $H$, et al. Radiation dosedependent hippocampal atrophy detected with longitudinal volumetric magnetic resonance imaging. Int J Radiat Oncol Biol Phys 2017;97:263-269.

31. Yi $H G$, Kim HJ, Kim YJ, et al. Epidermal growth factor receptor (EGFR) tyrosine kinase inhibitors (TKIs) are effective for leptomeningeal metastasis from non-small cell lung cancer patients with sensitive EGFR mutation or other predictive factors of good response for EGFR TKI. Lung Cancer 2009;65:80-4.

32. Lee $E_{1}$ Keam B, Kim DW, et al. Erlotinib versus gefitinib for control of leptomeningeal carcinomatosis in non-small-cell lung cancer. J Thorac Oncol 2013;8:1069-74.

33. Mok TS, Wu YL, Ahn MJ, et al. Osimertinib or platinumpemetrexed in EGFR T790M-positive lung cancer. N Engl J Med 2017;376:629-40.

34. Baik CS, Chamberlain MC, Chow LO. Targeted therapy for brain metastases in EGFR-mutated and ALK-rearranged non-smallcell lung cancer. J Thorac Oncol 2015;10:1268-78. 